\title{
A Two-Phase Hybrid Particle Swarm Optimization Algorithm for Solving Permutation Flow-Shop Scheduling Problem
}

\author{
Ko-Wei Huang \\ Institute of Computer and \\ Communication Engineering, \\ Department of Electrical \\ Engineering,National Cheng \\ Kung University, Tainan 70101, \\ Taiwan, ROC.
}

\author{
Chu-Sing Yang \\ Institute of Computer and \\ Communication Engineering, \\ Department of Electrical \\ Engineering,National Cheng \\ Kung University, Tainan 70101, \\ Taiwan, ROC.
}

\author{
Chun-Wei Tsai \\ Department of Applied \\ Geoinformatics, \\ Chia Nan University of \\ Pharmacy Science, Tainan \\ 71710, Taiwan, ROC
}

\begin{abstract}
In this paper, a two-phase hybrid particle swarm optimization algorithm (PRHPSO) is proposed for the permutation flowshop scheduling problem (PFSP) with the minimizing makespan measure. The smallest position value (SPV) rule is used for encoding the particles that enable PSO for suitable PFSP, and the NEH and Tabu search algorithms are used for initializing the particles. In the first phase, the pattern reduction (PR) operator is used in the $\mathrm{PSO}$ algorithm for reducing the computation time. In order to avoid a premature convergence, a regeneration operator is used for escaping to the local optimal and balancing exploitation and exploration in the second phase. Additionally, a simulated annealing (SA) algorithm is utilized for local search to improve the best solution after the PSO search process. Finally, the results show that PRHPSO is significantly faster than two PSO-based algorithms and presents large-sized benchmarks.
\end{abstract}

\section{General Terms}

Hybrid Metaheuristic Algorithm, Combinatorial optimization.

\section{Keywords}

Particle swarm optimization, Permutation flow-shop scheduling problem, Pattern reduction, Makespan, Pattern Reduction, Regeneration Operator.

\section{INTRODUCTION}

Johnson [1] pioneered permutation flow-shop problem research in 1953. Since then, the flow-shop scheduling problem (PFSP), an NP-hard problem, has received increasing attention in the combinatorial optimization research community. Rinnooy Kan [2] proved that minimizing the makespan was an NP-hard problem, and Garay et al. [3] proved that total flow-time minimization was also an NP-hard problem. Non-polynomial computing time is required for finding solutions. The objective of the PFSP is to find the sequence of jobs being processed in all machines to optimize the performance measure. Heuristics algorithms are commonly used for obtaining approximate solutions and decreasing CPU time in NP-hard combinatorial optimization problems. By 1983, the Nazwa-Enscore-Ham (NEH) [4] heuristics had been a well-known algorithm for makespan minimization for more than twenty-years.
Table 1: Examples permutation flowshop solution

\begin{tabular}{l|c}
\hline Solution & Redundant sub-solution \\
\hline 1234567 & \\
1235467 & \\
1236457 & 123 \\
1234765 & \\
1236574 & \\
\hline
\end{tabular}

The PFSP is defined as follows. Considering $n$ jobs $\left\{j_{1}, j_{2}, j_{3}, \ldots, j_{n}\right\}$ with each job successively processed by $m$ machines $\left\{M_{1}, M_{2}, M_{3}, \ldots, M_{m}\right\}$, the processing time is given by $p_{i, k}$ between job $i$ and machine $k$. On the other hand, there are rule restrictions in the PFSP that each machine is permitted processing one job at most, and each job can be processed on one machine at any time. The goal of the PFSP is to find the best sequence (order or permutation) of jobs and optimize the object function. There are two common object functions in PFSP, namely makespan and total flow-time minimization.It is given that $\pi=\left\{j_{1}, j_{2}, j_{3}, \ldots, j_{n}\right\}$, which indicates the job sequence, and $C\left(j_{i}, k\right)$, denotes the completion time of job $i$ on machine $k . C\left(j_{i}, k\right)$ can be defined as Eq. 1.

$$
\begin{aligned}
& C\left(j_{1}, 1\right)=p_{j_{1}, 1} \\
& C\left(j_{c}, 1\right)=C\left(j_{c-1}, 1\right)+p_{j_{c}, 1}, c=2,3, \ldots, n \\
& C\left(j_{1}, k\right)=C\left(j_{1}, k-1\right)+p_{j_{1}, k}, k=2,3, \ldots, m \\
& C\left(j_{c}, k\right)=\max \left\{C\left(j_{c-1}, k\right), C\left(j_{c}, k-1\right)\right\}+p_{j_{c}, k}, \\
& \quad \text { for } c=2,3, \ldots, n ; k=2,3, \ldots, m
\end{aligned}
$$

The objective of the makespan is to find permutation $\pi_{\text {best }}$ in permutation set $\Pi$, such that Eq. 2 shows:

$$
\pi_{\text {best }}=\underset{\forall \pi \in \Pi}{\operatorname{argmin}} C_{\max }(\pi) \text {, where } C_{\max }(\pi)=C\left(j_{n}, m\right)
$$

Meta-heuristic algorithms have been proposed for solving the PFSP and minimizing makespan. They include simulated annealing (SA) [5][6], Tabu search (TS) [7][8], genetic algorithms (GAs) [9][10], ant colony optimization (ACO) [11][12], differential evolution [13][14], and the hybrid (memetic) algorithm [15][16][17]. Among these algorithms, 
population-based heuristic approaches (such like PSO, GA, DE...etc.) all share the same critical problem when populations evolve over a period of time. For example, five solutions are obtained as shown in Table. 1. Each solution contains sub-solution 123 at the same position, meaning that it would spend redundant CPUcomputation time on calculating the makespan with sub-solution 123. If redundant calculations can be removed, the CPU computation time can be greatly reduced.This paper proposes a two-phaseparticle swarm optimization algorithm for solving permutation flow-shop scheduling problems. The most significant concerns are decreasing computation time and increasing the diversity of solutions. The algorithm has two tasks.

a) To eliminate redundant computations and reduce total computation time.

b) To enhance search behaviour and improve solution quality.

The task is divided into two parts. Common sub-solutions are detected and compressed in part 1 , while the regeneration operator is used for preserving exploitation and exploration when all solutions have the same job sequence in part 2. To improve the quality after the two-phase PSO algorithm, the SA-based local search is used for fine-tuning the solution, which is the simulated annealing (SA) [18] combined with the variable neighborhood local search [19]. The experimental results show that the algorithms are significantly faster than two recently proposed PSO-based algorithms using a different scale of benchmarks.

The remainder of this paper is organized as follows. Background knowledge and related work are described in Section 2. Section 3 states the details of the proposed algorithms. Evaluations of the proposed algorithms are described in Section 4. Finally, conclusions and future work are described in Section 5.

\section{BACKGROUND AND RELATED WORK}

In this section, the background of this research field and the related study are reviewed. The overview will focus on four main topics of

a) NEH heuristic algorithm (NEH),

b) Tabu Search (TS),

c) Simulated Annealing (SA), and

d) Particle Swarm Optimization (PSO).

\subsection{NEH Heuristic algorithm}

For more than, the Nawaz-Enscore-Ham (NEH) [4] heuristic algorithm has a widely-used algorithm for solving the makespan minimization problem. The NEH algorithm is divided into three parts.

1. Each job is sorted in descending order of processing time on the machines.

2. The first two jobs are chosen. A better partial order is obtained by scheduling them according to a comparison of makespan values.

3. Job $j=3, \ldots, \mathrm{n}$, is inserted at each of $j$ 's possible positions in the sequence to obtain the best partial schedule which minimizes the partial makespan.

Although the NEH heuristics is easy to implement, its time complexity is $O\left(n^{3} m\right)$. Many studies have proposed ways to improve the quality or computation time of the original NEH heuristic, such as [20][21][22].

\subsection{Tabu Search}

Tabu (also called Taboo) search (TS), which was proposed by Glover et al. [23], is a meta-heuristic algorithm used for combinatorial optimization problems. The motivation for TS comes from the visited solutions and repeated visits of local search approaches. TS creates a short-memory structure that records forbidden moves called a tabu list. The foundation of tabu search is described as follows. First, the initial solution is generated randomly. Second, a set of neighborhood (candidate) solutions is generated using the current solution. Third, the solution with the best admissibility is chosen (the solution with the best admissibility is the one in which the move satisfies the aspiration criterion) and the tabu list is updated. Finally, Steps 2 and 3 are repeated until the stopping criterion is reached.

\subsection{Simulated Annealing}

The idea for the annealing process originated in metalwork. When metal is heated to a high temperature, it becomes a liquid. As a liquid, the structure changes and, when it cools, it re-solidifies in different shapes. Inspired by Metropolis et al. [24] and physical annealing, simulated annealing (SA) was proposed by Kirkpatrick et al. [18]. It became a well-known heuristic algorithm and has been used for twenty years. SA is a very applicable heuristic algorithm for combinatorial problems that uses a neighborhood searching strategy. SA begins from a random initial solution. A new state is generated using a perturbation method. The change in energy is expressed by Eq. 3 .

$$
\Delta E=f(\text { new })-f(\text { old })(3)
$$

wheref (new) is the new state object value and $f($ old $)$ is the current best solution value. The new state is accepted if $\Delta E<0$. If $\Delta E>0$, the new state may still be accepted if the Boltzmann distribution is satisfied with Eq. 4 .

$$
\text { Prob }<\left(e^{-\frac{\Delta E}{T}}\right)
$$

where Prob is a random number $[0,1]$ and $T$ is a dynamic control parameter. The SA process stops when it freezes or the set number of iterations is reached.

\subsection{Particle Swarm Optimization}

Particle swarm optimization (PSO) is a population-based swarm intelligence algorithm that was first presented by Kennedy and Eberhard in 1995 [25], when the original PSO was used for continuous optimization (i.e., function optimization). PSO was first used for discrete problem [26] in 1997; the first article discussing the application of PSO to solving PFSP was presented by Tasgetiren et al. [27] in 2004. Some study applied particle swarm optimization (PSO) to solving the PFSP, such as [28][29][30][31][32][33][34][35][36].The main idea is that each individual updates its current solution to reference with its own history experiences and the experiences of others. Base on [25] and [37], each particle is generated using a random solution. The goal for each particle is to search for solution space and update its own solution between cognitive behaviour and social behaviour in the swarm, as the following Eqs.5 and 6.

$$
\begin{array}{r}
v_{i, j}^{t+1}=\omega v_{i, j}^{t}+c_{1} r_{1}\left(p_{i, j}^{t}-x_{i, j}^{t}\right)+c_{2} r_{2}\left(p_{g b e s t, j}^{t}-x_{i, j}^{t}\right)(5) \\
x_{i, j}^{t+1}=x_{i, j}^{t}+v_{i, j}^{t+1}(6)
\end{array}
$$




\begin{tabular}{|c|c|}
\hline Output: & $\begin{array}{l}\text { Swarm size } N \text {, parameters } \omega, c_{1} \text {, and } c_{2} \text {, } \\
\text { and maximum number of iterations } t_{\max } \\
\text { Best solution } x\end{array}$ \\
\hline Step: & Description: \\
\hline 1. & $\begin{array}{l}\text { Randomly initialize the position and ve- } \\
\text { locity in the search space. }\end{array}$ \\
\hline 2. & Evaluate each particle \\
\hline 3. & $\begin{array}{l}\text { Find the personal best } p_{i} \text {, where } i= \\
1,2, \ldots, N\end{array}$ \\
\hline 4. & Find the global best $p_{\text {gbest }}$ \\
\hline 5 . & $\begin{array}{l}\text { Update the position and velocity of each } \\
\text { particle according to Eq. } 5 \text { and } 6\end{array}$ \\
\hline 6. & $\begin{array}{l}\text { Go back to Step } 2 \text { until } t_{\max } \text { is reached. } \\
\text { Return the best solution } x \text {. }\end{array}$ \\
\hline
\end{tabular}

Fig 1: A standard particle swarm optimization (PSO) algorithm

Table 2: Examples representing particle $i$

\begin{tabular}{cc|cc}
\hline Position j & Position value & Ranking of values & Job order \\
\hline 1 & -0.43 & -2.98 & 3 \\
2 & 3.14 & -2.37 & 7 \\
3 & -2.98 & -0.43 & 1 \\
4 & 2.61 & 0.25 & 6 \\
5 & 1.56 & 1.56 & 5 \\
6 & 0.25 & 2.61 & 4 \\
7 & -2.37 & 3.14 & 2 \\
\hline
\end{tabular}

Table 3: Position information examples obtained from the permutation solutions

\begin{tabular}{c|cc|c}
\hline Position j & $\begin{array}{c}\text { NEH/TS } \\
\text { solution }\end{array}$ & Random value & $\begin{array}{c}\text { Mapping } \\
\text { value }\end{array}$ \\
\hline 1 & 3 & -3.14 & -0.43 \\
\hline 2 & 7 & -2.37 & 3.14 \\
\hline 3 & 1 & -0.43 & -2.98 \\
\hline 4 & 6 & 1.56 & 2.61 \\
\hline 5 & 5 & 0.25 & 1.56 \\
\hline 6 & 4 & 2.61 & 0.25 \\
\hline 7 & 2 & -2.98 & -2.37 \\
\hline
\end{tabular}

where $d$ is the dimension of the search area and tisthe iteration number. The position and velocity of particle $i$ in iteration $t$ are $X_{i}^{t}=\left\{X_{i, 1}^{t}, X_{i, 2}^{t}, X_{i, 3}^{t}, \ldots, X_{i, d}^{t}\right\} \quad$ and $V_{i}^{t}=\left\{V_{i, 1}^{t}, V_{i, 2}^{t}, V_{i, 3}^{t}, \ldots, V_{i, d}^{t}\right\}$, respectively. Personal best $p_{i}^{t}=\left\{p_{i, 1}^{t}, p_{i, 2}^{t}, p_{i, 3}^{t}, \ldots, p_{i, d}^{t}\right\}$ corresponds to particle $\mathrm{i}$ and is the best fitness value so far during time period t. The global best is respected so that $p_{\text {gbest }, j}^{t}=\left\{p_{\text {gbest }, 1}^{t}, p_{\text {gbest }, 2}^{t}, p_{\text {gbest }, 3}^{t}, \ldots, p_{\text {gbest }, d}^{t}\right\}, \quad$ which indicates the best fitness solution found since initialization. Inertia weight $\omega$ is used for controlling the convergence speed, $c_{1}$ is the acceleration weight cognitive element, $c_{2}$ is the weight of the social parameter, and $r_{1}$ and $r_{2}$ are random numbers uniformly distributed in the area of $[0,1]$. The personal best update processing of particle $i$ and the current best (global best) solution in the swarm is updated using the minimizing the object function $f$.

Figure 1 show the procedure for the standard PSO approach.

\section{THE PROPOSED ALGORITHM}

This PRHPSO algorithm consists of two main phases during the PSO evolution, namely the pattern reduction operator phase and regeneration operator phase. This section introduces pattern reduction and the regeneration operator, which are adapted into the PSO-based algorithm for solving the permutation flow-shop scheduling problem (PFSP).

\subsection{Particle Encoding}

In the PSO algorithm, the particles fly through the solution space engaging in social behaviour. Encoding particles in the population for the PFSP is an important issue. In order to find a suitable direct mapping between the positions (dimensions) of particles and the job sequence, this paper employs a heuristic approach called smallest position value (SPV) [38], which is used for converting the continuous values into permutations of the job sequence. In short, each particle is represented by $\mathrm{n}$ continuous values. In the decoding process, these continuous values are transformed into a permutation of the job sequence by the SPV rule. Table 2 illustrates the process of decoding a particle using the SPV rule.

The second column denotes the position values of the particle. The fourth column indicates the increase of each value. According to the SPV value, -2.98 is the smallest position value. The first job order in $x_{i, 1}$ is 3 . Then, -2.37 is picked and $x_{i, 2}$ is assigned in 7 . The remaining values are picked according to their ranking to construct the permutation. Thus, the complete job permutation $\pi=(3,7,1,6,5,4,2)$.

\subsection{Particle Initialization}

To ensure that the initial swarm has a quality solution, the NEH [4] heuristic algorithm and TS [23] are applied to generating two solutions. The remaining particles are given random values (including position and velocity) using

$$
\begin{aligned}
& x_{i, j}=x_{u, b} * r \\
& v_{i, j}=v_{l, b}+\left(v_{u, b}-v_{l, b}\right) * r
\end{aligned}
$$

where $x_{u, b}=5.0, v_{l, b}=-5.0, v_{u, b}=5.0$, and $r$ is in the interval $[0,1]$. Table 3 shows an example of position values generated from the permutation results.

\subsection{Pattern Reduction}

In the first phase, the concept of the pattern reduction (PR) technique was presented by Chiang et al. [39]. It was used for solving a clustering problem. According to previous research, the PR can be used in meta-heuristics algorithms for solving the combinatorial optimization problem.

In this paper, we modify the basic PR for the PFSP. This operator is divided into two parts, namely detection and compression. The goal of PR is to quickly eliminate redundant process during PSO processing. Details of the two parts are given below. Before the PR phase, the current best solution will be called parent 1 and used in regeneration operator phases.

\subsubsection{Detection operator:}

In this operator, the solutions are inspected against all positions (dimensions), which are marked as either undetected or detected, (detected positions are indicated by 1 , others by $0)$. The detection vector is defined as.

$$
D_{\text {vec }} \in(\{0,1\}, \forall \text { positions })
$$


Table 4: Rearrangement the of permutationusing newposition value and spv rule

\begin{tabular}{c|c|c|c}
\hline $\begin{array}{c}\text { (Iteration i-1) } \\
\text { Position j }\end{array}$ & $\begin{array}{c}\text { Position } \\
\text { value }\end{array}$ & $\begin{array}{c}\text { Ranking of } \\
\text { values }\end{array}$ & $\begin{array}{c}\text { Job } \\
\text { order }\end{array}$ \\
\hline 1 & -0.43 & -2.98 & 3 \\
2 & 3.14 & -2.37 & 7 \\
3 & -2.98 & -0.43 & 1 \\
4 & 2.61 & 0.25 & 6 \\
5 & 1.56 & 1.56 & 5 \\
6 & 0.25 & 2.61 & 4 \\
7 & -2.37 & 3.14 & 2 \\
\hline \multicolumn{3}{|c}{} \\
\hline (Iteration i) & Position & Ranking of & Job \\
Position j & value & values & order \\
\hline 1 & 1.26 & -2.01 & 4 \\
2 & -0.72 & -1.67 & 7 \\
3 & 1.68 & -0.72 & 2 \\
4 & -2.01 & 0.53 & 5 \\
5 & 0.53 & 1.26 & 1 \\
6 & 3.91 & 1.68 & 3 \\
7 & -1.67 & 3.91 & 6 \\
\hline
\end{tabular}

Table 5: Rearrangement of position values after the pattern reduction operator

\begin{tabular}{c|c|c}
\hline $\begin{array}{c}\text { (Iteration i) } \\
\text { Position j }\end{array}$ & Job order & Position value \\
\hline 1 & 4 & 1.26 \\
2 & 7 & -0.72 \\
3 & 2 & 1.68 \\
4 & 5 & -2.01 \\
5 & 1 & 0.53 \\
6 & 3 & 3.91 \\
7 & 6 & -1.67 \\
\hline
\end{tabular}

\begin{tabular}{c|c|c}
\hline $\begin{array}{c}\text { (After PR) } \\
\text { Position j }\end{array}$ & Job order & Position value \\
\hline 1 & 47251 & -2.14 \\
2 & 3 & 1.68 \\
3 & 6 & 3.91 \\
\hline
\end{tabular}

Table 1 shows that all of the five permutation solutions have three detected positions $(1,2$, and 3$)$, which have respective element values of $\{1\},\{2\}$, and $\{3\}$. The following rules are used for determining the trigger condition for the compression operator.

i.) If there are fewer than two positions marked as detected.

ii.) If there are at least two positions labelled detected, but none is marked as connected.

iii.) If there are two or more elements marked as connected.

If the solutions belong to casei.) or case ii.), the compression part is not triggered.

\subsubsection{Compression operator:}

In this part, the connected positions can be readily obtained from common sub-solutions by interacting with the detection vector. If the connective sub-solutions detected are redundant, they can be merged into one element. Considering the previous example in this operator, elements $\{1\},\{2\}$, and $\{3\}$ can be merged into one set $\{123\}$. The $\{123\}$ may find the connective sub-solution $\{64\}$ and the two sub-solutions may be combined into one set $\{12364\}$. Then the set $\{12364\}$ will be compressed into one element $\{1\}$. Finally, the solution will become $\{157\}$, which means only the makespan of the job sequence $\{157\}$ needs to be calculated, rather than $\{1236457\}$.

\subsection{Particle Updating}

A particle moves in a direction according to the inertia weight. The best position and the best position so far of other particles can be formalized using Eqs.5 and 6. These two equations are used for determining the final position of particle $i$ in iteration $t$. However, new velocity and position values are obtained aftereachiteration. Thus, after updating particle position values, the permutation jobs sequence must be rearranged by the SPV rule. Table 4 shows how to determine the new permutation sequence after particle update. Table 5 shows another example of how to determine and reduce the dimensions of the new position values after the PR operator. It can be seen in Table 5 that the job sequence $\{4725136\}$ will be reduced and compressed into the new job sequence $\{436\}$. Thus the PR can reduce the computation time.

After the PR operator, we can get the convergence solution, parent 2. Although the PR approach can reduce the computation time, it may cause particles to converge very quickly. Thus, the regeneration operator method is used for improving the quality and diversity of the PSO algorithm.

\subsection{Regenerate Operator}

The concept in this phase uses the crossover operator - order operator [40] $(\mathrm{OX})$ to reconstruct all particles by using parent 1 and parent 2 . The use of parent 1 , parent 2 and (OX) operator should be used for reconstructing the remaining particles. Fig. 2 shows how the regeneration operator rebuilds the remaining particles. After the reconstruction phase, each new particle (solution) is used for reinitializing the position and velocity values according to the SPV rule. This new population will be evaluated by using the PSO algorithm until the termination criteria are reached, but without employing the PR operator again.

\subsection{SA-based Local Search}

In order to add diversity and improve quality, four neighborhood perturbation methods (SA-based VNS local search) can be used in the SA local search, namely pair-swap, insertion, inversion, and displacement.

a) Pair-Swap: Randomly select two distinct positions and swap them.

b) Insertion: Randomly select two distinct positions (a, b) and insert $b$ in front of $a$.

c) Inversion: Invert the subsequence between two random positions in the solution.

d) Displacement: Select a subsequence and one cut point, and insert in the subsequence before the cut point position. Figure 3 shows a simple example illustrating each of the neighborhood perturbation methods. 


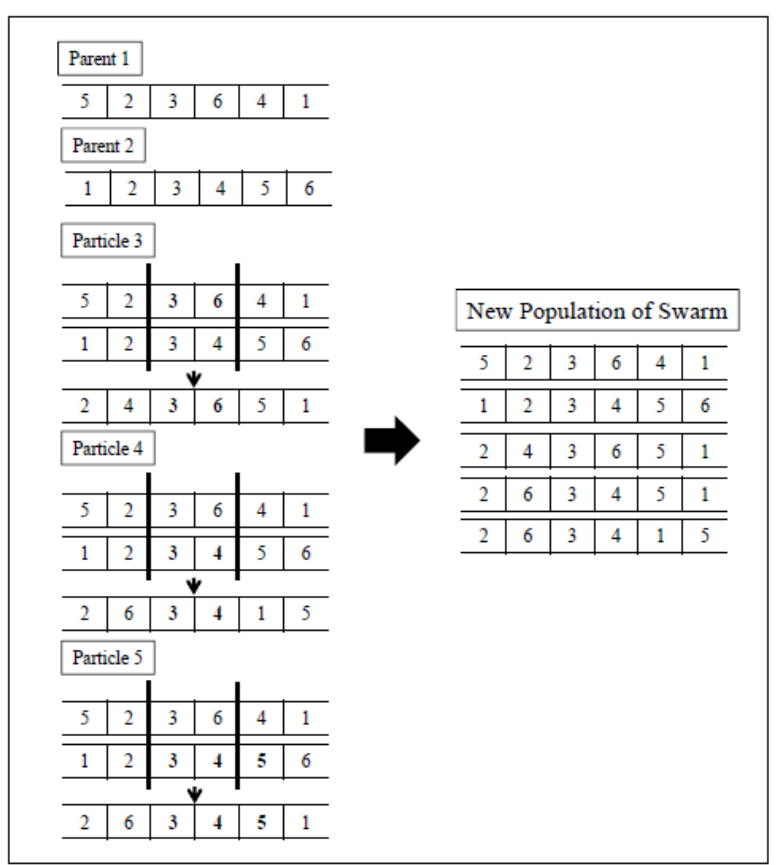

Fig 2: Example regeneration operator.

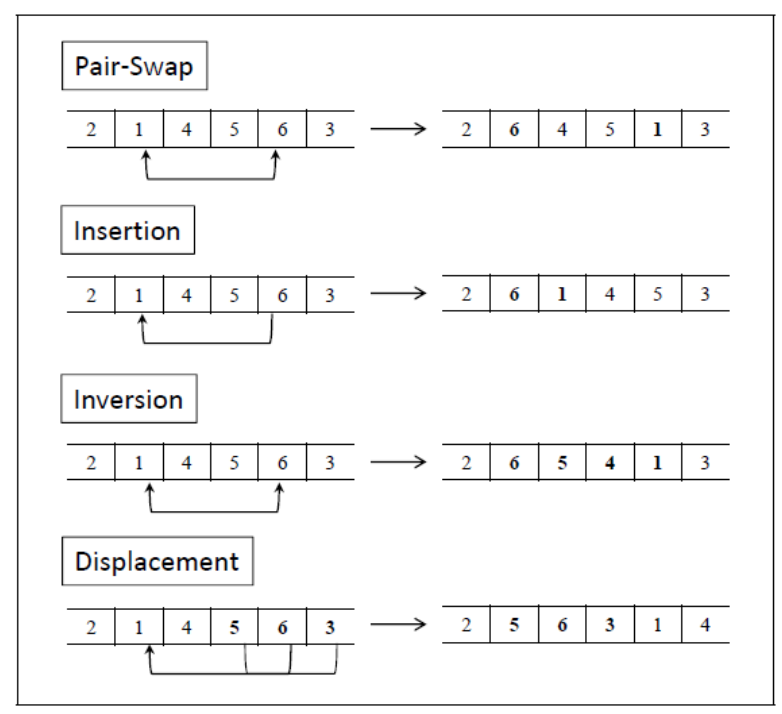

Fig 3: Example of the perturbation methods.

\subsection{The PRHPSO algorithm}

The PRHPSO algorithm is a two-phase hybrid PSO-based algorithm for solving PFSP. Phase 1 uses the PR technique to reduce computation time and, in phase 2 , a regeneration operator is adopted to balance exploitation and exploration and restart the PSO evolution process without using the PR operator.

Finally, the SA-based local search preserves the best solution after the regeneration operator.The PRHPSO algorithm is illustrated in Fig. 4.

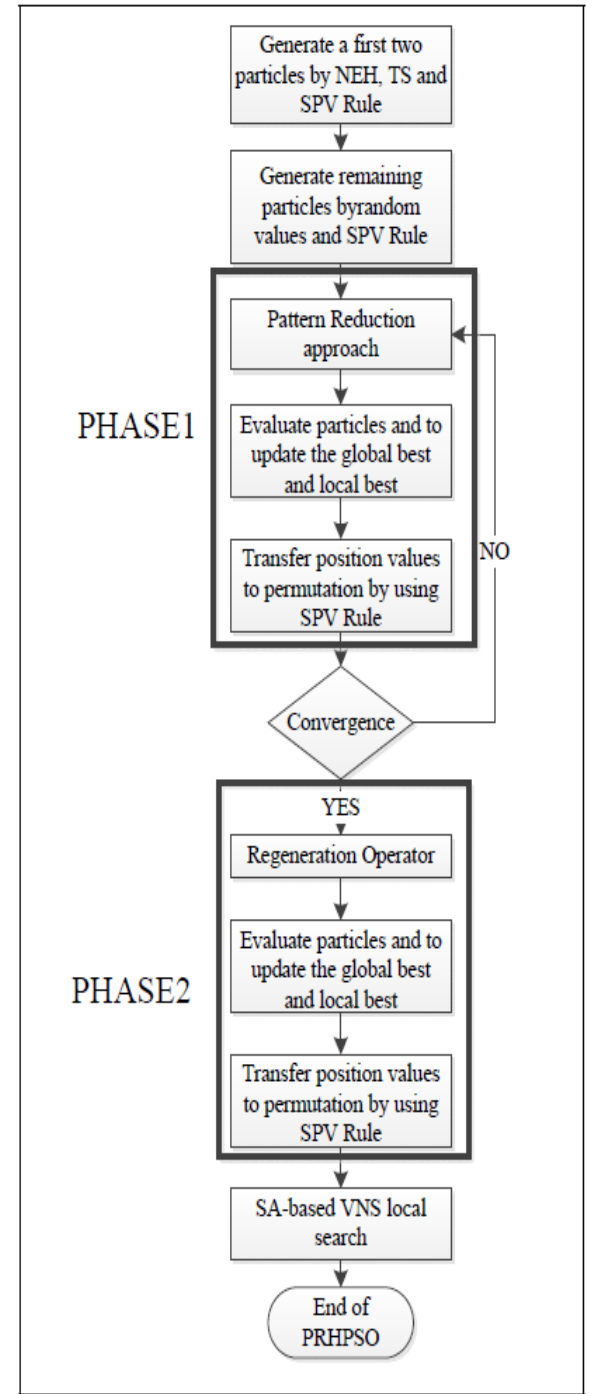

Fig 4: The flowchart of the PRHPSO.

\subsection{An Example}

Considering the five solutions in Table 1, the process for executing PRHPSO for this example is shown in Fig. 5. The PR and regeneration operator are applied to the PSO convergence processing. Assuming that the current best solution sequence (parent 1) is $\{523641\}$ before the PR operator, and parent 2 is $\{123456\}$ at the PR operator, then parent 1 and parent 2 pass through the regeneration operator OX to reconstruct the remaining particles. Straightforward the PSO process, the best solution becomes $\{251364\}$. Finally, the best solution $\{251364\}$ is adjusted to $\{314562\}$ using the SA-based local search.

\section{EXPERIMENTAL RESULTS}

In this section, the performance of the proposed algorithm is evaluated. The PRHPSO refers to the approach of applyingthe PSO with PR to reduce the computation time, regenerate the particle population, balance intensification and diversification, and fine-tune the SA-based local search. The PRHPSO is compared to two algorithms; one is the swap-based algorithm [28] and the other is the SPV-based algorithm [32]. 


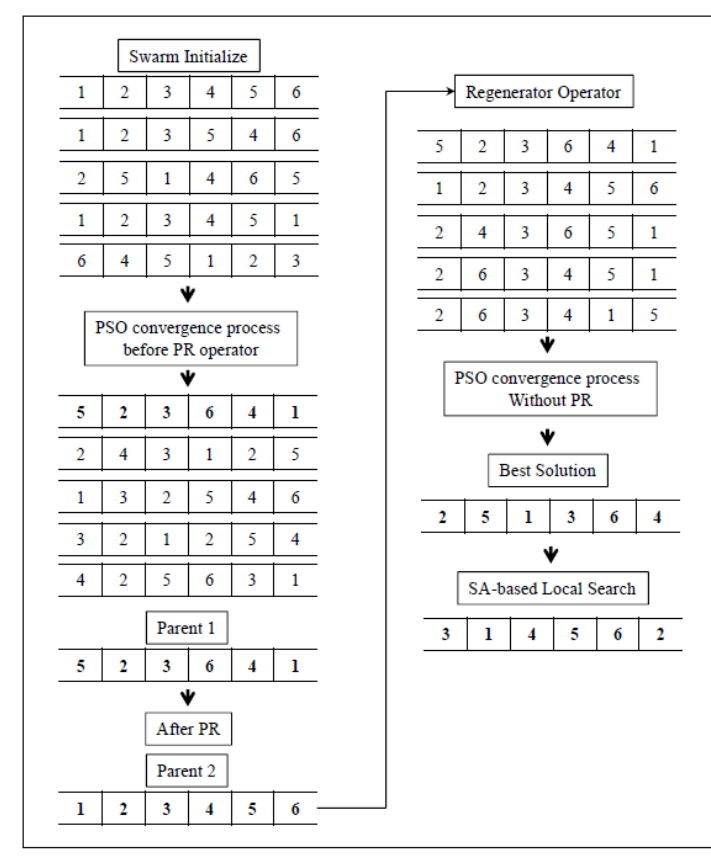

5: An Example of PRHPSO algorithm.

Table 6: The Taillard benchmarks for permutation flowshop problem

\begin{tabular}{cc}
\hline Name of instance & $\mathbf{n}$ jobs $* \mathbf{m}$ machines \\
\hline Ta01 & $20 * 5$ \\
Ta11 & $20 * 10$ \\
Ta21 & $20 * 20$ \\
Ta31 & $50 * 5$ \\
Ta41 & $50 * 10$ \\
Ta51 & $50 * 20$ \\
Ta61 & $100 * 5$ \\
Ta71 & $100 * 10$ \\
Ta81 & $100 * 20$ \\
Ta91 & $200 * 10$ \\
Ta101 & $200 * 20$ \\
Ta111 & $500 * 20$ \\
\hline
\end{tabular}

Table 7Parameter settings for permutation flow-shop instance

\begin{tabular}{c|c}
\hline PSO parameters & \\
\hline Swarm size & 50 \\
\# of iterations & 1000 \\
PR start iteration & 20 \\
\# of simulations (R) & 30 \\
\# of Regeneration & 100 \\
Operator iterators & \\
$\omega$ & 0.9 to 0.4 \\
$c_{1}, c_{2}$ & 2 \\
\hline SA parameters & 100 \\
\hline Initial temperature T & 0.99 \\
Cooling coefficient $\beta$ & \\
\hline TS parameters & 7 \\
\hline Size of Tabu list & 1000 \\
\# of iterations &
\end{tabular}

Table 8Experimental results of the PRHPSO against DPSO

\begin{tabular}{ccccc}
\hline \multirow{2}{*}{ Name of instance } & \multicolumn{2}{c}{ DPSO $[28]$} & \multicolumn{2}{c}{ PRHPSO } \\
\cline { 2 - 5 } & ARPD & $t_{\text {avg }}$ & ARPD & $t_{\text {avg }}$ \\
\hline Ta01 & 0.94 & $\mathbf{0 . 4 0}$ & $\mathbf{0 . 0 6}$ & 0.78 \\
Ta11 & 4.74 & $\mathbf{0 . 4 7}$ & $\mathbf{0 . 6 9}$ & 0.92 \\
Ta21 & 4.13 & $\mathbf{0 . 6 3}$ & $\mathbf{0 . 5 2}$ & 0.73 \\
Ta31 & 0.77 & $\mathbf{1 . 7 6}$ & $\mathbf{0 . 1 8}$ & 2.36 \\
Ta41 & 6.15 & $\mathbf{1 . 8 8}$ & $\mathbf{2 . 8 0}$ & 2.65 \\
Ta51 & 4.05 & $\mathbf{2 . 2 7}$ & $\mathbf{2 . 9 3}$ & 3.22 \\
Ta61 & 0.33 & 6.23 & $\mathbf{0 . 0 4}$ & $\mathbf{5 . 9 4}$ \\
Ta71 & 2.01 & 6.42 & $\mathbf{0 . 9 2}$ & $\mathbf{5 . 7 7}$ \\
Ta81 & 3.72 & 7.27 & $\mathbf{2 . 0 8}$ & $\mathbf{6 . 2 9}$ \\
Ta91 & 15.74 & 23.35 & $\mathbf{0 . 6 9}$ & $\mathbf{1 7 . 8 6}$ \\
Ta101 & 3.01 & 25.68 & $\mathbf{2 . 2 1}$ & $\mathbf{2 1 . 0 3}$ \\
Ta111 & 7.47 & 142.93 & $\mathbf{1 . 9 7}$ & $\mathbf{1 0 9 . 7 6}$ \\
\hline Average & 4.82 & 19.94 & $\mathbf{1 . 3 7}$ & $\mathbf{1 6 . 1 2}$ \\
\hline
\end{tabular}

Table 9: Experimental results of the PRHPSO against PSOVNS

\begin{tabular}{ccccc}
\hline \multirow{2}{*}{ Name of instance } & \multicolumn{2}{c}{ DPSO $[28]$} & \multicolumn{2}{c}{ PRHPSO } \\
\cline { 2 - 5 } & ARPD & $t_{\text {avg }}$ & ARPD & $t_{\text {avg }}$ \\
\hline Ta01 & $\mathbf{0 . 0 3}$ & 9.84 & 0.06 & $\mathbf{0 . 7 8}$ \\
Ta11 & $\mathbf{0 . 0 4}$ & 13.93 & 0.69 & $\mathbf{0 . 9 2}$ \\
Ta21 & $\mathbf{0 . 1 8}$ & 21.57 & 0.52 & $\mathbf{0 . 7 3}$ \\
Ta31 & $\mathbf{0 . 0 2}$ & 22.37 & 0.18 & $\mathbf{2 . 3 6}$ \\
Ta41 & $\mathbf{1 . 7 3}$ & 30.56 & 2.80 & $\mathbf{2 . 6 5}$ \\
Ta51 & $\mathbf{2 . 1 2}$ & 46.80 & 2.93 & $\mathbf{3 . 2 2}$ \\
Ta61 & 0.06 & 43.72 & $\mathbf{0 . 0 4}$ & $\mathbf{5 . 9 4}$ \\
Ta71 & $\mathbf{0 . 4 5}$ & 59.43 & 0.92 & $\mathbf{5 . 7 7}$ \\
Ta81 & $\mathbf{1 . 5 7}$ & 92.89 & 2.08 & $\mathbf{6 . 2 9}$ \\
Ta91 & 0.76 & 121.70 & $\mathbf{0 . 6 9}$ & $\mathbf{1 7 . 8 6}$ \\
Ta101 & $\mathbf{1 . 4 9}$ & 190.49 & 2.21 & $\mathbf{2 1 . 0 3}$ \\
Ta111 & $\mathbf{1 . 1 7}$ & 522.22 & 1.97 & $\mathbf{1 0 9 . 7 6}$ \\
\hline Average & $\mathbf{0 . 8 8}$ & 106.95 & 1.37 & $\mathbf{1 6 . 1 2}$ \\
\hline
\end{tabular}

The dataset generated by Taillard [41] was used in this evaluation. The well-known benchmark composed of 120 instances from 20 jobs and 5 machines (Ta01) to 500 jobs and 20 machines (Ta111) for permutation flow-shop scheduling, as shown in Table VI. All experiments were performed on a computer with a core(TM)2 Quad Q9400 2.66GHz Intel CPU with $2 \mathrm{~GB}$ of memory running on Microsoft Windows 7 . In the experiments, all of the programs were implemented using Java language. The PSO, SA, and TS parameter settings are shown in Table VII. Table VIII and Table IX show a comparison of the simulation results. The average percentage relative deviation ARPD is shown in Eq. 10 and the average computation time is denoted ast $t_{\text {avg }}$ seconds.

$$
\operatorname{ARPD}=\sum_{i}^{R}\left(\left(\frac{Q_{i}-Q_{b k s}}{Q_{b k s}}\right) * 100 \%\right) / \mathrm{R}
$$

where $Q_{i}$ denotes the values of the makespan of algorithm found, $Q_{b k s}$ indicates the best known solutions, and $\mathrm{R}$ denotes the number of simulations. The performance can be divided into the two parts, the ARPD value and the computation time. 
Table VIII and Table IX show the results of the DPSO, PSOVNS, and the proposed PRHPSO algorithms for the best known solutions for the Taillard's benchmark. In Table VIII, the PRHPSO is slightly slower than the DPSO in the dataset $\mathrm{Ta} 01$ to Ta61. This is because the DPSO does not have the SPV-rule mapping and rearranging strategy. By using the SPV-rule, regeneration operator and SA-based local search, the PRHPSO outperforms the DPSO in ARPD measure. In Table IX, the PRHPSO is faster than the PSOVNS almost 6 times in total average; except in Ta61 and Ta91, it losses quality in ARPD. This is because the PR operator leads the PSO to fast convergence, and regeneration operator and SA increase the quality.

\section{CONCLUSIONS}

A two-phase mememic PSO algorithm (PRHPSO) is proposed for solving the permutation flow-shop scheduling problem. The experimental results indicate that the proposed method is faster than other algorithms with regard to CPU time in large scale of benchmarks and has some quality loss compared to PSOVNS. Many things can be done to refine this algorithm. (1) More effective detection strategies should be developed for the pattern reduction phase of algorithm. (2) In making the ARPD solutions better than those of PSOVNS or other effective algorithms, another powerful regeneration approach should be considered, meaning to enhance the solution searching abilities of particles.

\section{ACKNOWLEDGMENTS}

This work was supported in part by the National ScienceCouncil, Taiwan, R.O.C., under grants NSC 1002218-E-006-031-MY3.

\section{REFERENCES}

[1] S. M. Johnson, "Optimal two- and three-stage production schedules withsetup times included," Naval Research Logistics Quarterly, vol. 1, pp.61-68, 1954.

[2] A. H. G. Rinnooy Kan, Machine scheduling problems: Classification,complexity and computations. Martinus Nijhoff, The Hague, 1976.

[3] M. R. Garey, D. S. Johnson, and R. Sethi, "The complexity of flowshopand jobshop scheduling," Mathematics of Operations Research, vol. 1,pp. 117129, 1976.

[4] M. Nawaz, E. E. Enscore Jr, and I. Ham, "A heuristic algorithm for them-machine, n-job flow-shop sequencing problem," OMEGA, vol. 11,no. 1, pp. 91-95, 1983.

[5] I. H. Osman and C. N. Potts, "Simulated annealing for permutationflow-shop scheduling," Omega, vol. 17, no. 6, pp. 551-557, 1989.

[6] C. Low, J. Yeh, and K. Huang., "A robust simulated annealing heuristicfor flow shop scheduling problem," International Journal of AdvancedManufacturing Technology, vol. 23, pp. 762-767, 2004.

[7] E. Nowicki and C. Smutnicki, "A fast tabu search algorithm for thepermutation flow-shop problem," European Journal of OperationalResearch, vol. 91, no. 1, pp. 160-175, 1996.

[8] J. P.Watson, L. Barbulescu, L. D. Whitley, and A. E. Howe, "Contrastingstructured and random permutation flow-shop scheduling problems:Search-space topology and algorithm performance," ORSA Journal onComputing, vol. 14, pp. 98-123, 2002.

[9] C. R. Reeves, "A genetic algorithm for flowshop sequencing," Computersand Operations Research., vol. 22, pp. 5-13, January 1995.

[10] O. Etiler, B. Toklu, M. Atak, and J. Wilson., "A genetic algorithmfor flow shop scheduling problems," The Journal of the OperationalResearch Society, vol. 55, no. 8, pp. 830-835, 2004.

[11] T. St utzle, F. Intellektik, F. Informatik, and T. H. Darmstadt, "Anant approach to the flow shop problem," in In Proceedings of the6th European Congress on Intelligent Techniques \& Soft Computing(EUFIT'98, 1997, pp. 1560-1564.

[12] C. Rajendran and H. Ziegler, "Ant-colony algorithms for permutationflowshop scheduling to minimize makespan/total flowtime of jobs,"European Journal of Operational Research, vol. 155, no. 2, pp. 426438,2004

[13] Q.-K. Pan, M. F. Tasgetiren, and Y.-C. Liang, "A discrete differentialevolution algorithm for the permutation flowshop scheduling problem,"Computers and Industrial Engineering, vol. 55, no. 4, pp. 795816,2008

[14] Q.-K. Pan, L. Wang, and B. Qian, "A novel differential evolution algorithmfor bi-criteria no-wait flow shop scheduling problems," Computersand Operations Research, vol. 36, pp. 2498-2511, 2009.

[15] B. Qian, L. Wang, R. Hu, W.-L. Wang, D.-X. Huang, and X. Wang, "Ahybrid differential evolution method for permutation flow-shop scheduling,"The International Journal of Advanced Manufacturing Technology,vol. 38, pp. $757-777,2008$

[16] Y. Zhang, X. Li, and Q. Wang, "Hybrid genetic algorithm for permutationflowshop scheduling problems with total flowtime minimization,"European Journal of Operational Research, vol. 196, no. 3, pp. 869-876,2009.

[17] T.-C. Chiang, H.-C. Cheng, and L.-C. Fu, "NNMA: An effective memeticalgorithm for solving multiobjective permutation flow shop schedulingproblems," Expert Systems with Applications, vol. 38, no. 5, pp. 5986 5999, 2011..

[18] S. Kirkpatrick, C. D. Gelatt, and M. P. Vecchi, "Optimization bysimulated annealing," Science, vol. 220, pp. 671-680, 1983.

[19] N. Mladenovic, "Variable neighborhood search," Computers and OperationsResearch, vol. 24, no. 11, pp. 1097-1100, 1997.

[20] E. Taillard, "Some efficient heuristic methods for the flow shop sequencingproblem," European Journal of Operational Research, vol. 47, no. 1,pp. 65-74, Jul. 1990.

[21] T. Aldowaisan and A. Allahverdi, "New heuristics for no-wait flowshopsto minimize makespan," Computers and Operations Research, vol. 30,no. 8, pp. 1219 - 1231, 2003.

[22] P. J. Kalczynski and J. Kamburowski, “An improved neh heuristicto minimize makespan in permutation flow 
shops," Computers andOperations Research, vol. 35, pp. 3001-3008, 2008.

[23] F. Glover and M. Laguna, Tabu Search. Norwell, MA, USA: KluwerAcademic Publishers, 1997.

[24] N. Metropolis, A. W. Rosenbluth, M. N. Rosenbluth, A. H. Teller, andE. Teller, "Equation of State Calculations by Fast Computing Machines,"The Journal of Chemical Physics, vol. 21, no. 6, pp. 1087-1092, 1953.

[25] J. Kennedy and R. Eberhart, "Particle swarm optimization," in NeuralNetworks, 1995. Proceedings., IEEE International Conference on, vol. 4,1995, pp. 1942-1948.

[26] J. Kennedy and R. C. Eberhart, "A discrete binary version of the particleswarm algorithm," in IEEE International Conference on Systems, Man,and Cybernetics,, vol. 5, 1997, pp. 4104-4108.

[27] M. Tasgetiren, M. Sevkli, Y.-C. Liang, and G. Gencyilmaz, "Particleswarm optimization algorithm for permutation flowshop sequencingproblem," in Ant Colony Optimization and Swarm Intelligence. SpringerBerlin / Heidelberg, 2004.

[28] K. Rameshkumar, R. Suresh, and K. Mohanasundaram, "Discrete particleswarm optimization (dpso) algorithm for permutation flowshopscheduling to minimize makespan," in Advances in Natural Computation,ser. Lecture Notes in Computer Science. Springer Berlin /Heidelberg, 2005, vol. 3612, p. 572581.

[29] Z. Lian, X. Gu, and B. Jiao, "A similar particle swarm optimizationalgorithm for permutation flowshop scheduling to minimize makespan,"Applied Mathematics and Computation, vol. 175, pp. 773-785, 2006.

[30] C.-J. Liao, C.-T. Tseng, and P. Luarn, "A discrete version of particleswarm optimization for flowshop scheduling problems," Computers andOperations Research, vol. 34, pp. 3099-3111, 2007.

[31] B. Liu, L. Wang, and Y.-H. Jin, "An effective pso-based memeticalgorithm for flow shop scheduling," Systems, Man, and Cybernetics,Part B: Cybernetics, IEEE Transactions on, vol. 37, no. 1, pp. 18 -27,feb. 2007.

[32] M. F. Tasgetiren, Y.-C. Liang, M. Sevkli, and G. Gencyilmaz, "A particleswarm optimization algorithm for makespan and total flowtime minimizationin the permutation flowshop sequencing problem," EuropeanJournal of Operational Research, vol. 177, no. 3, pp. $1930-1947,2007$.

[33] C. Zhang, J. Sun, X. Zhu, and Q. Yang, “An improved particle swarmoptimization algorithm for flowshop scheduling problem," InformationProcessing Letters, vol 108, pp. 204-209, 2008.

[34] D. Sha and H. Hung Lin, "A particle swarm optimization for multiobjectiveflowshop scheduling," The International Journal of AdvancedManufacturing Technology, vol. 45, pp. 749-758, 2009.

[35] C. Zhang, J. Ning, and D. Ouyang, "A hybrid alternate two phases particleswarm optimization algorithm for flow shop scheduling problem,"Computers and Industrial Engineering, vol. 58, pp. 1-11, February 2010.

[36] H. Liu, L. Gao, and Q. Pan, "A hybrid particle swarm optimization withestimation of distribution algorithm for solving permutation flowshopscheduling problem," Expert Systems with Applications, vol. 38, pp.4348 4360, 2011

[37] Y. Shi and R. Eberhart, "A modified particle swarm optimizer," in Proceedingsof IEEE international conference on evolutionary computation,1998, pp. 6973 .

[38] J. C. Bean, "Genetic algorithms and random keys for sequencing andoptimization," ORSA Journal on Computing, vol. 6, no. 2, pp. 154-160,1994.

[39] M.-C. Chiang, C.-W. Tsai, and C.-S. Yang, "A timeefficient patterreduction algorithm for k-means clustering," Inf. Sci., vol. 181, no. 4,pp. 716-731, Feb. 2011.

[40] I. M. Oliver, D. J. Smith, and J. R. C. Holland, "A study of permutationcrossover operators on the traveling salesman problem," in Proceedingsof the Second International Conference on Genetic Algorithms onGenetic algorithms and their application. Hillsdale, NJ, USA: L.Erlbaum Associates Inc., 1987, pp. 224-230.

[41] E. Taillard, "Benchmarks for basic scheduling problems," EuropeanJournal of Operational Research, vol. 64 , no. 2, pp. 278-285, January1993. 